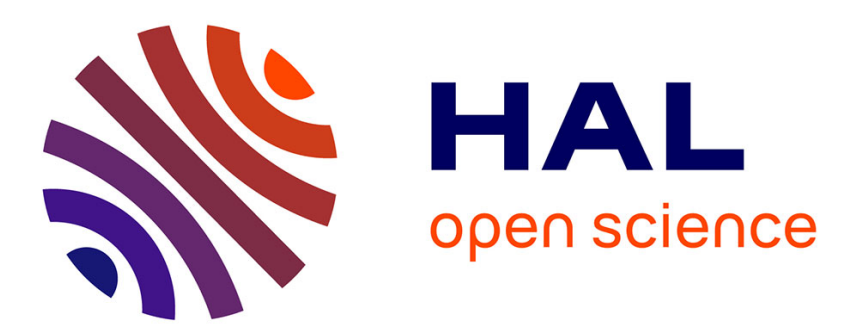

\title{
Thermal fluctuations of dislocations reveal the interplay between their core energy and long-range elasticity
}

\author{
Pierre-Antoine Geslin, David Rodney
}

\section{To cite this version:}

Pierre-Antoine Geslin, David Rodney. Thermal fluctuations of dislocations reveal the interplay between their core energy and long-range elasticity. Physical Review B: Condensed Matter and Materials Physics (1998-2015), 2018, 98 (17), 10.1103/PhysRevB.98.174115 . hal-02110637

\section{HAL Id: hal-02110637 \\ https://hal.science/hal-02110637}

Submitted on 7 May 2019

HAL is a multi-disciplinary open access archive for the deposit and dissemination of scientific research documents, whether they are published or not. The documents may come from teaching and research institutions in France or abroad, or from public or private research centers.
L'archive ouverte pluridisciplinaire HAL, est destinée au dépôt et à la diffusion de documents scientifiques de niveau recherche, publiés ou non, émanant des établissements d'enseignement et de recherche français ou étrangers, des laboratoires publics ou privés. 


\title{
Thermal fluctuations of dislocations reveal the interplay between their core energy and long-range elasticity
}

\author{
Pierre-Antoine Geslin* \\ ELyTMaX UMI 3757, CNRS - Université de Lyon - Tohoku University, International Joint Unit, Tohoku University, Sendai, Japan; \\ Institute for Materials Research, Tohoku University, 2-1-1 Katahira, Aobaku, Sendai 980-8577, Japan; \\ and Frontier Research Institute for Interdisciplinary Sciences, Tohoku University, Aramaki, Aoba 6-3, Aobaku, Sendai 980-8578, Japan \\ David Rodney ${ }^{\dagger}$ \\ Univ. Lyon, Université Claude Bernard Lyon 1, CNRS, Institut Lumière Matière, LYON, F-69622 France
}

(Received 21 May 2018; revised manuscript received 6 November 2018; published 29 November 2018)

\begin{abstract}
Equilibrium fluctuations of dislocations are central to the plastic response of metals and alloys because they control the attempt frequency of thermally activated events. We analyze here atomic-scale simulations of thermally vibrating dislocations with the help of an analytical model and show that thermal fluctuations intimately involve both long-range elasticity and short-range core effects. In addition, equilibrium fluctuations of edge and screw dislocations in aluminum are used to derive quantitative parameters that characterize their energetics and dynamics and we discuss how large-scale models such as dislocation dynamics can be parametrized based on these results. In particular, we show that the core parameters found here through fluctuations are transferable and can be used to predict dislocation bow-out under an applied stress.
\end{abstract}

DOI: 10.1103/PhysRevB.98.174115

\section{INTRODUCTION}

Dislocations are linear defects responsible for the plastic flow of metals and alloys. While an important body of work has focused on their athermal properties (core energy, lattice friction, elastic interactions, stress-induced curvature, etc.) [1-4], their behavior at finite temperatures remains only partially explored. While recent progress was obtained on the free energy barrier against dislocation motion [5,6], dislocation thermal vibrations remain poorly understood despite their importance in setting the attempt frequency of thermally activated dislocation processes such as dislocation movement in solution-strengthened alloys, kink-pair nucleation, and cross slip $[7,8]$.

Dislocation vibrations were the subject of the seminal works of Friedel [9] and Granato and Lücke [10], who modeled a dislocation based on the simplifying assumption that it behaves like a vibrating string characterized by a line tension $\Gamma$ and an energy $E=\Gamma \ell$, with $\ell$ the dislocation length. At thermal equilibrium, the mean square amplitude of a vibrational mode is then inversely proportional to the square of its wave vector $k$ (see, e.g., Ref. [11]), a consequence of the equipartition theorem. Such dependence was measured using molecular dynamics (MD) on fluctuating solid/liquid interfaces [12]. Using this so-called capillary fluctuation method, the analysis of solid/liquid interfacial fluctuations has been used to assess the solid/liquid anisotropy $[12,13]$ and the

\footnotetext{
*pierre-antoine.geslin@insa-lyon.fr

†david.rodney@univ-lyon1.fr
}

kinetics coefficient [14], necessary to parametrize quantitatively phase-field models of solidification [15]. In contrast, it was shown that thermal fluctuations of grain boundaries follow a different scaling because of the long-range elastic interactions induced by their fluctuations $[16,17]$.

As for dislocations, a line tension model accounts only for short-range effects and ignores their long-range elastic interactions. The latter may affect the vibrational spectrum and hence the attempt frequency of thermally activated events [18]. In this paper, we use face centered cubic (FCC) aluminum as a benchmark and study from the atomic scale the vibrational spectrum of edge and screw dislocations. We find a $1 / k^{2}$ dependence but only at short wavelengths where core effects dominate, while a different scaling in $1 /\left[k^{2} \log \left(k_{0} / k\right)\right]$ is obtained at long wavelengths due to long-range elasticity, in accordance with previous works $[19,20]$. The spectra can be fully explained by modeling core effects with a line tension and elasticity with the nonsingular theory of dislocations of Cai et al. [21]. This theory avoids the core singularity by spreading the Burgers vector over a finite distance called the core parameter. Fitting the vibrational spectra against our analytical model allows us to extract character-dependent core parameters and line tension coefficients. The reliability and transferability of these parameters are demonstrated by reproducing dislocation bow-out under an applied shear stress. Moreover, analyzing the time-correlations of the vibrations, we obtain wavelength-dependent mass and damping coefficient for both dislocation characters. Use of these parameters in mesoscale dislocation dynamics (DD) models [22-24] as well as in recent attempts to model thermal fluctuations of crystalline defects as Langevin forces [25] is discussed. 

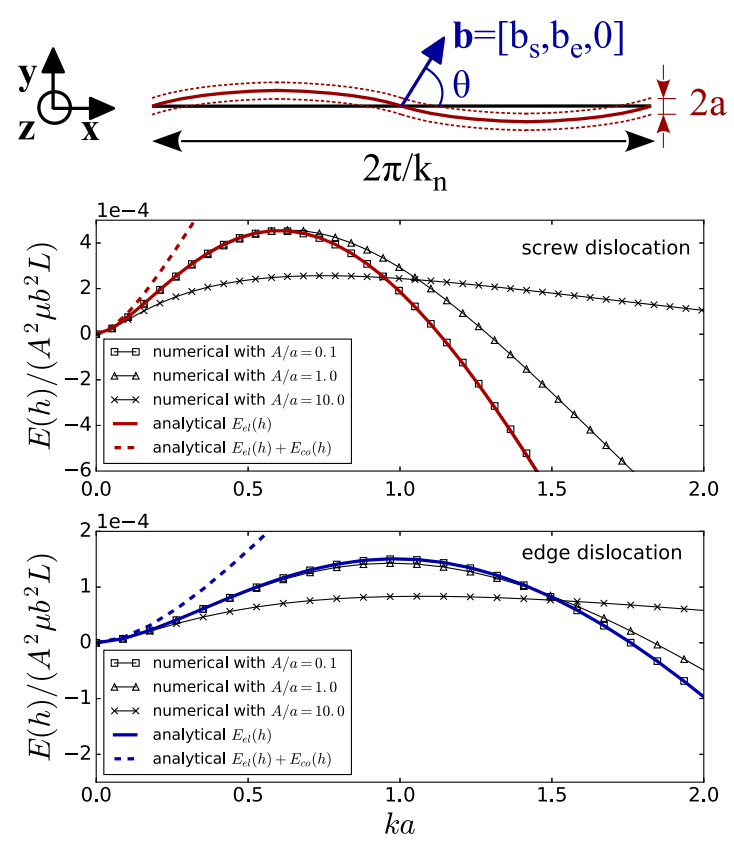

FIG. 1. Normalized elastic energy of a sinusoidal perturbation on screw and edge dislocations compared to numerical calculations (thin lines with symbols). The total energy $E_{e l}+E_{c o}$ is shown as dashed lines for values of the line tension reported in Table II.

\section{ENERGY OF A PERTURBED DISLOCATION}

We consider an infinite dislocation line of Burgers vector $\left[b_{s}, b_{e}, 0\right]$ (see Fig. 1) and a core parameter $a$ that represents the core spreading distance in the nonsingular dislocation theory [21]. We assume a perturbation $h(x), L$ periodic in the $x$ direction, expressed as a Fourier series $h(x)=$ $\sum_{n=-N_{\max }}^{N_{\max }} C_{n} e^{i k_{n} x}$ where $k_{n}=2 \pi n / L$ and $C_{-n}=C_{n}^{*}[h(x)$ is real]. We do not consider fluctuations out of the glide plane because at large wavelengths, they would involve climb [26] and at small wavelengths, they would correspond to line tension terms independent of the in-plane fluctuations. Within isotropic elasticity, and assuming that the perturbation varies slowly [i.e., $\forall x, h^{\prime}(x) \ll 1$ ], the excess self-energy due to the perturbation can be expressed analytically within the nonsingular theory of dislocations as

$$
E_{e l}(h)=\frac{L}{2} \sum_{n=1}^{N_{\max }} \mathcal{K}_{e l}\left(k_{n}\right)\left|C_{n}\right|^{2}
$$

with $\mathcal{K}_{e l}$ an elastic stiffness expressed as:

$$
\begin{aligned}
\mathcal{K}_{e l}(k)= & \frac{\mu}{\pi(1-v) a^{2}}\left\{-\left(2 b_{s}^{2}(1-v)+b_{e}^{2}\right)\right. \\
& +a^{2} k^{2} K_{0}(a k)\left(2 b_{s}^{2}-v b_{e}^{2}\right)+a k \\
& \times K_{1}(a k)\left[(3-v) b_{s}^{2}-b_{e}^{2}\left(1-\frac{a^{2} k^{2}(1-v)}{2}\right)\right] \\
& \left.-a^{2} k^{2} K_{2}(a k)\left(\frac{b_{s}^{2}(1+v)}{2}-b_{e}^{2}\right)\right\},
\end{aligned}
$$

where $\mu$ and $v$ denote the shear modulus and Poisson ratio, and $K_{i}(i=0,1,2)$ are modified Bessel functions of the second kind (see Sec. 1 of Ref. [27] for details of the calculations). We note that this analytical solution assumes isotropic elasticity. Generalizing our approach to anisotropic elasticity would require the use of a numerical approach (see, e.g., Refs. [24,28]).

In addition to the elastic contribution, we account for the core energy of the perturbation using a line tension model. As shown previously [2,29,30], this contribution allows us to account for the additional energy due to the nonlinearities in the core region that cannot be accounted for with linear elasticity. The energy $E_{c o}(h)$ has the same form as Eq. (1) and a stiffness (see Sec. 2 of Ref. [27])

$$
\mathcal{K}_{c o}(k)=2 \Gamma k^{2} \text {. }
$$

To assess the domain of validity of Eqs. (1)-(2), we consider a sinusoidal perturbation $h(x)=A \sin (k x)$. We compute the elastic energy in Eq. (1) and compare with a numerical estimate obtained from the interaction energy between straight segments of the discretized dislocation line [21]. We consider here a dislocation length $L=400 \AA$ with elastic parameters of aluminum $(v=0.34, \mu=29.8 \mathrm{GPa})$. As shown in Fig. 1, the numerical solutions converge towards the prediction of Eqs. (1)-(2) in the limit of small amplitude $A$ or small wave vector $k$. It is worth mentioning that the elastic energy of a screw dislocation grows faster with $k$ than for an edge dislocation due the different nature of elastic interactions. We also note that for large wave vectors, the elastic energy becomes negative. This occurs for $k a \gtrsim 1$, which is when the wavelength of the fluctuations becomes of the order of the core parameter, i.e. in a region where spreading the Burgers vector may lead to artifacts [31]. The potential instability associated with a negative elastic energy is, however, counterbalanced by the core energy in Eq. (3) (see dashed lines in Fig. 1), which increases as $k^{2}$ and far exceeds the elastic energy at large $k$.

Vibrational spectra are predicted from the dislocation energy using the equipartition theorem. Adding both the core and elastic contributions yields the total energy of a perturbed dislocation, expressed in the same form as in Eq. (1) but with the total stiffness $\mathcal{K}=\mathcal{K}_{e l}+\mathcal{K}_{c o}$. Since the energy is the sum of quadratic contributions of independent degrees of freedom (two terms per Fourier mode because $C_{n}$ is complex), the equipartition theorem ensures that at thermal equilibrium:

$$
\left\langle\left|C_{n}\right|^{2}\right\rangle=\frac{2 k_{B} T}{L \mathcal{K}\left(k_{n}\right)} .
$$

\section{MOLECULAR DYNAMICS SIMULATIONS AND POWER SPECTRA}

In this section, we compare the prediction of Eq. (4) with MD simulations. We chose FCC aluminum because of its near isotropic elasticity and the availability of a reliable interatomic potential [32]. For both screw and edge characters, a dislocation dipole is initially introduced in a large simulation cell through its displacement field $[2,29,33,34]$. The position of the dislocations in the cell was chosen such that the interactions between the dislocations of the dipole and their periodic images is reduced to a minimum (see Fig. 2). The dimensions of the periodic cells reported in Table I were selected to keep comparable dislocation lengths and interdislocation distances 

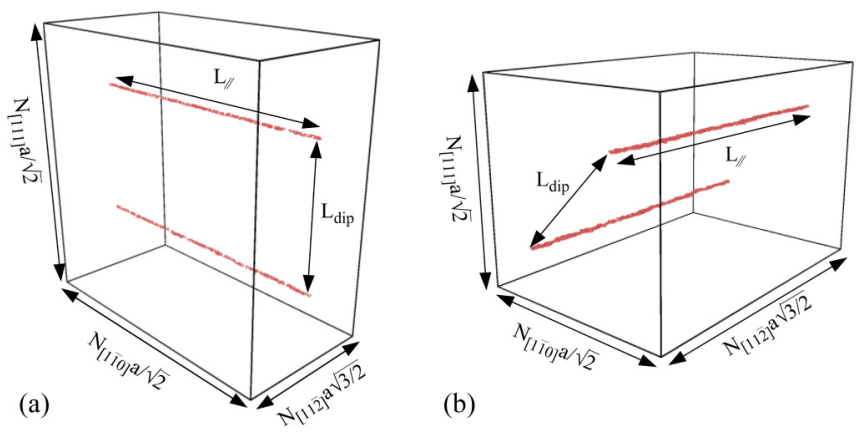

(b)

FIG. 2. Visualization of the simulation cell used for the (a) screw and (b) edge dislocations (only the atoms having a HCP stacking that belong to the stacking fault are shown).

for both characters. We then performed long MD simulations with LAMMPS [35] in the microcanonical (NVE) ensemble to generate a large number of dislocation configurations.

The dislocation position was estimated using the common neighbor analysis (CNA) implemented in LAMMPS: the atoms belonging to the stacking fault between the two partials are detected as having a hexagonal (HCP) stacking. To improve the estimate of the dislocation position, we also include the atoms of the partial dislocation core defined as defective FCC atoms, nearest neighbors of the stacking fault atoms. The position of the dislocation is then obtained by averaging the positions of the stacking fault and core atoms in equispaced bins along the dislocation line.

We discuss here results obtained at $300 \mathrm{~K}$, but we checked that our methodology also applies at other temperatures (see Sec. 5 of Ref. [27]). From ten independent 0.2 ns long simulations, we extract about 20000 dislocation configurations, and compute the vibrational spectra from their Fourier transform. Figure 3 displays the resulting spectra for edge and screw characters.

We first note that the power spectrum of the edge dislocation is significantly higher than that of the screw, as expected from the lower energy of a perturbation on an edge than on a screw dislocation seen in Fig. 1. Two regimes can be distinguished with a transition at around $k \sim 0.5 \AA^{-1}$, (i.e., a wavelength $\sim 13 \AA$ ): large $k$ fluctuations are dominated by the core contribution and scale approximately as $1 / k^{2}$, while small $k$ fluctuations show a different scaling and are dominated by the elastic energy. As expected from previous works $[19,20]$, an expansion of Eq. (2) in the limit $a k \ll 1$ shows a $1 /\left[k^{2} \log \left(k_{0} / k\right)\right]$ scaling displayed in Fig. 3.

Furthermore, fitting the power spectra with Eq. (4) allows us to determine the best choice of core parameter $a$ and line tension $\Gamma$ for both orientations. The fits were performed in the central part of the spectra (full lines in Fig. 3) because the long

TABLE I. Dimensions of the molecular dynamics simulation cells.

\begin{tabular}{lcccccc}
\hline \hline character & $N_{[1 \overline{1} 0]}$ & $N_{[11 \overline{2}]}$ & $N_{[111]}$ & $N_{\text {atoms }}$ & $L_{\|}[\AA]$ & $L_{\text {dip }}[\AA]$ \\
\hline screw & 173 & 50 & 70 & 3633000 & 495.4 & 245.5 \\
edge & 120 & 100 & 48 & 3441600 & 496.0 & 240.6 \\
\hline \hline
\end{tabular}

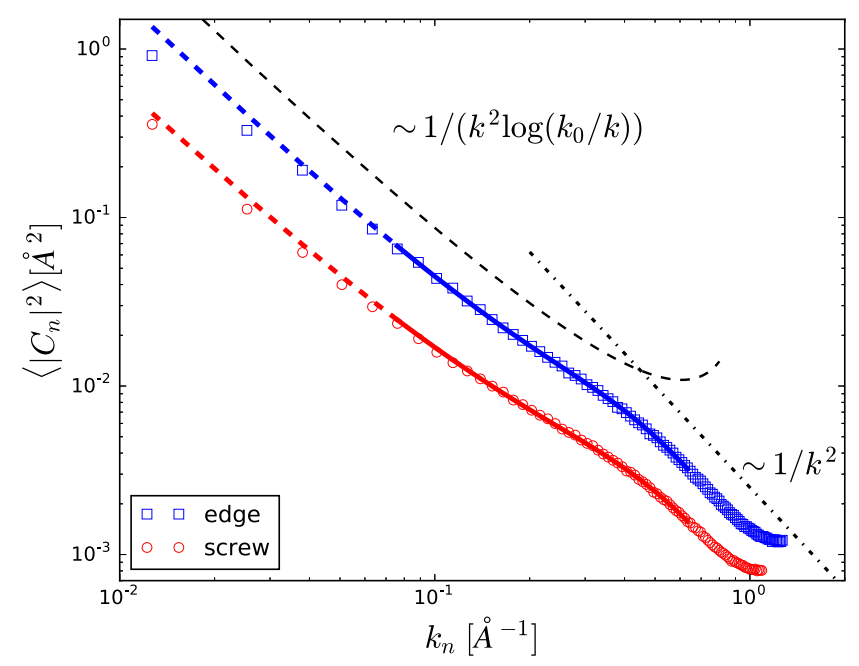

FIG. 3. Vibrational spectra obtained at $300 \mathrm{~K}$ for screw and edge dislocations (symbols) and corresponding fits (lines) from Eq. (4). Fluctuation amplitudes are averaged over a large number of configurations such that uncertainties are smaller than the symbol size. The dashed lines are guides for the eye representing asymptotic behaviors in the limit of small and large wave vectors.

and short wavelength fluctuations are affected, respectively, by long-range interactions with periodic images and the discrete nature of the crystalline structure. The fits are of high quality, showing that dislocation fluctuations can be faithfully reproduced using the analytical model of Eq. (4).

Table II lists the parameters $a$ and $\Gamma$ obtained from fitting the power spectra obtained at $300 \mathrm{~K}$. We also report here the line tension computed at $0 \mathrm{~K}$ from static energy calculations on straight dislocations (see Ref. [24] and Sec. 4 of Ref. [27]). For straight dislocations, the choice of $a$ is arbitrary $[4,24]$ and the $0 \mathrm{~K}$ line tensions in Table II were computed for the same $a$ value as found at $300 \mathrm{~K}$. By way of contrast, the present calculations yield a unique pair $(a, \Gamma)$ for each orientation because for fluctuating curved dislocations, $a$ defines uniquely the wavelength where long-range elasticity ceases to dominate over core effects. We added in Table II the dissociation distance $d_{0}$ of edge and screw dislocations to highlight that $a$ increases with $d_{0}$. The values of $a$ obtained here therefore capture the larger physical spreading of the edge dislocation core compared to the screw. In addition, the line tensions deduced from the fluctuation spectra are in good agreement with the ones obtained at $0 \mathrm{~K}$.

TABLE II. Fitted values of the core parameters and line tension obtained at $300 \mathrm{~K}$ and comparison with dissociation distances and line tensions obtained from molecular static simulations.

\begin{tabular}{llc}
\hline \hline character & screw & edge \\
\hline$a$ (from fit) [̊] & 3.34 & 5.60 \\
$d_{0}(0 \mathrm{~K})[\AA]($ from $[24])$ & 6.34 & 12.19 \\
$\Gamma$ (from fit) $[\mathrm{eV} / \AA]$ & 0.114 & 0.053 \\
$\Gamma_{a}(0 \mathrm{~K})[\mathrm{eV} / \AA]$ & 0.134 & 0.085 \\
\hline \hline
\end{tabular}



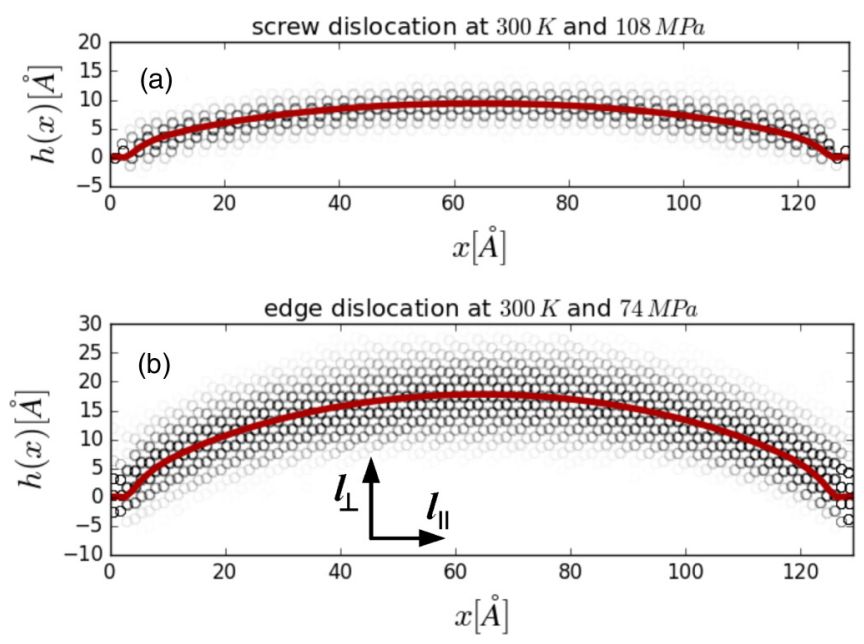

FIG. 4. Comparison between bow-out obtained from atomistic calculations (atoms belonging to the stacking fault are shown) and from the elastic model (continuous red line).

\section{TRANSFERABILITY TO DISLOCATION BOW-OUT}

To check the reliability of our results and in analogy with the fluctuation-dissipation theorem, we investigated the bow-out of periodically pinned dislocations under a small external stress, as considered in the past by Szajewski et al. [4]. We considered as above dipoles of either edge or screw dislocations but with a smaller length $L \approx 130 \AA$. A resolved shear stress was applied by straining the simulation cells. The dislocations are pinned by freezing the atoms located within a distance $\bar{d}=b$ of the sides of simulation cell and belonging to the dislocation core. Reasonable stresses below $110 \mathrm{MPa}$ are considered to remain in the limit of a small bow-out.

Figure 4 displays the averaged atomistic configurations obtained for both screw and edge dislocations at $300 \mathrm{~K}$.

To compare these atomistic results with our analytical approach, we use the energy of a deformed dislocation discussed in Sec. II. We use the fact that the dislocation shape is symmetric with respect to $L / 2$ (see Fig. 4) to express the dislocation shape as a sum of cosine functions:

$$
h_{b o}(x)=A_{0}+\sum_{n=1}^{N_{\max }} A_{n} \cos \left(k_{n} x\right),
$$

where $A_{0}$ is the average height of the dislocation. To enforce the fact that the dislocation is pinned, we impose $h(x=0)=0$ by the condition $A_{0}=-\sum_{n=1}^{N_{\max }} A_{n}$. The energy related to the bow-out is given by Eqs. (1)-(3) with $C_{-n}=$ $C_{n}=A_{n} / 2$ and an extra term to account for the work of the Peach-Koehler force:

$$
\begin{aligned}
E_{p k} & =-A_{0} L\left[\left(\boldsymbol{\sigma}^{a} \boldsymbol{b} \wedge \boldsymbol{l}_{\|}\right) \cdot \boldsymbol{l}_{\perp}\right] \\
& =-A_{0} L f_{p k} \\
& =L f_{p k} \sum_{n=1}^{N_{\max }} A_{n},
\end{aligned}
$$

where $\boldsymbol{l}_{\|}$and $\boldsymbol{l}_{\perp}$ are unit vectors lying in the slip plane of the dislocation, respectively, parallel and perpendicular to the straight dislocation line (see Fig. 4). In addition, to enforce the fact that the dislocation position remains fixed on a length $\bar{d}$ close to the sides of the cell, we add a Lagrangian multiplier $\mathcal{L}\left(\left\{A_{n}\right\}\right)$ to the total energy to enforce $h(\bar{d})=h(L-\bar{d})=0$. Therefore, the energy of the dislocation is expressed as a function of the coefficients $A_{n}$ :

$$
\begin{aligned}
E_{\mathrm{tot}}\left(\left\{A_{n}\right\}\right)= & E_{e l}\left(\left\{A_{n}\right\}\right)+E_{c o}\left(\left\{A_{n}\right\}\right) \\
& +E_{p k}\left(\left\{A_{n}\right\}\right)+\mathcal{L}\left(\left\{A_{n}\right\}\right) .
\end{aligned}
$$

The equilibrium configuration of the dislocation is obtained by minimizing this total energy with respect to the amplitudes $A_{n}$ using a conjugate gradient algorithm.

Figure 4 shows the comparison between atomistic results and elastic calculations for both screw and edge characters with parameters taken from Table II. We first notice the very good agreement between the molecular dynamics results and elastic calculations for both characters. Slight differences can be seen near the obstacles, due to the difficulty to impose perfectly equivalent boundary conditions in atomistic and the elastic models. On the other hand, the curvature of the dislocations is practically identical far from the blocked atoms. Therefore, the parameters deduced from the analysis of fluctuations at finite temperature can be directly used to predict quantitatively bow-out configurations under finite stresses.

\section{TIME-DEPENDENT BEHAVIOR}

The dynamical behavior of the dislocations can be further investigated by computing the time correlation of the vibrational modes, $\left\langle C_{n}(0) C_{n}^{*}(t)\right\rangle$ in connection with Langevin models of defect dynamics [25]. In this case, the time correlations should decrease following underdamped dynamics:

$$
\left\langle C_{n}(0) C_{n}^{*}(t)\right\rangle=\left\langle\left|C_{n}\right|^{2}\right\rangle \cos \left(\omega_{n} t\right) e^{-t / \tau_{n}},
$$

where the coefficients $\omega_{n}$ and $\tau_{n}$ are related to the effective mass $M_{n}$ and drag coefficient $B_{n}$ of the Langevin equation [36] (see Sec. 3 of Ref. [27] for details). Figures 5(a) and 5(b) confirm that the time correlations exhibit the expected underdamped behavior for the screw and edge characters. Using nonlinear fits, we obtained the drag coefficient and effective mass displayed in Figs. 5(c) and 5(d). We note that the fits are of better quality for the edge than for the screw dislocation. We attribute this to the larger Peierls stress of the screw (not accounted for in the present approach), that slows down its dynamics and affects the fluctuation kinetics.

As expected from dislocation theory [1], the drag coefficient of the screw dislocation is larger than the edge for any wave vector. Figures 5(c) and 5(d) also demonstrate that the drag coefficient and effective mass depend significantly on the wave vector, showing that perturbations are dumped more quickly for small wavelengths due to the increase of $B$ and decrease of $M$ with $k$. In particular, the drag coefficient of the edge dislocation depends linearly on the wave vector and extrapolates to $B_{0}=13.4 \mu \mathrm{Pa} / \mathrm{s}$ in the limit of infinite wavelengths, close to the value obtained by Bitzek [37,38] for straight dislocations. By way of contrast, the effective mass does not depend significantly on the dislocation character and decreases roughly exponentially with the wave vector [see dashed line in Fig. 5(d)]. Using this crude fit, the extrapolation to a straight line yields $M_{0}=48 \mathrm{fg} / \mathrm{m}$, again close to the 

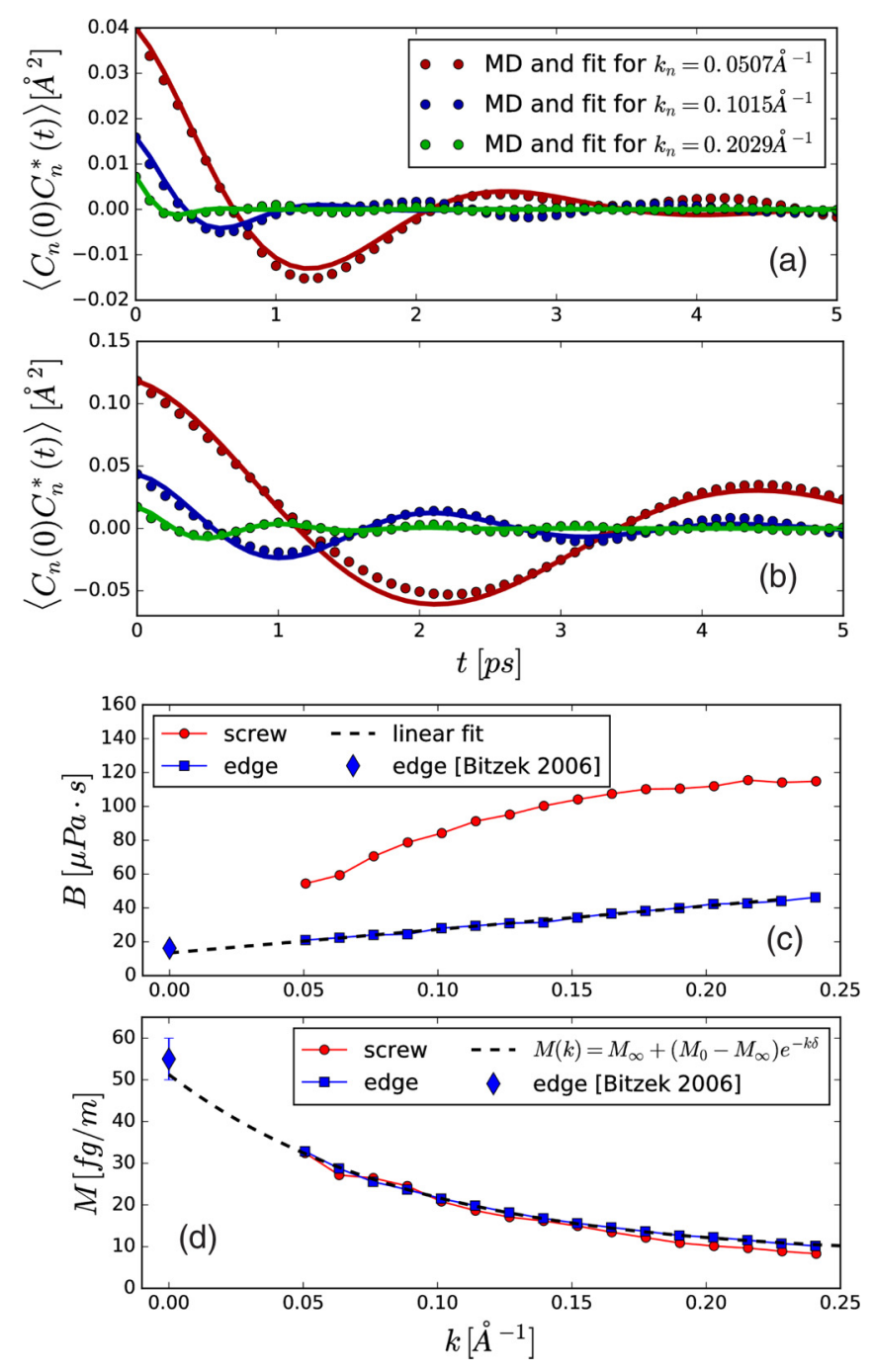

FIG. 5. Examples of time correlation functions for the (a) screw and (b) edge dislocation. (c) Drag coefficient and (d) effective mass are shown as a function of wave vector and compared with data obtained for a straight edge dislocation [37].

value reported by Bitzek (with a different method and at $30 \mathrm{~K}$ ) $[37,38]$.

\section{EXTRACTING PARAMETERS FOR HIGHER-SCALE MODELS}

In Sec. III, we have shown that reproducing accurately the power spectra obtained from atomistic simulations requires different core parameters for the edge and screw characters (see Table II). This character dependence reflects the change of core structure with dislocation orientation and the particular difficulty to represent dissociated dislocations with perfect, albeit spread, dislocations. However, the nonsingular theory and its numerical implementation in DD codes require a single character-independent value of $a[21,22,39,40]$.

To obtain parameters compatible with the DD formalism, the power spectra can also be fitted at long wavelengths by enforcing a single value of $a$ as shown in Fig. 6(a). We then obtain a core parameter $a=3.50 \AA$ and line tensions
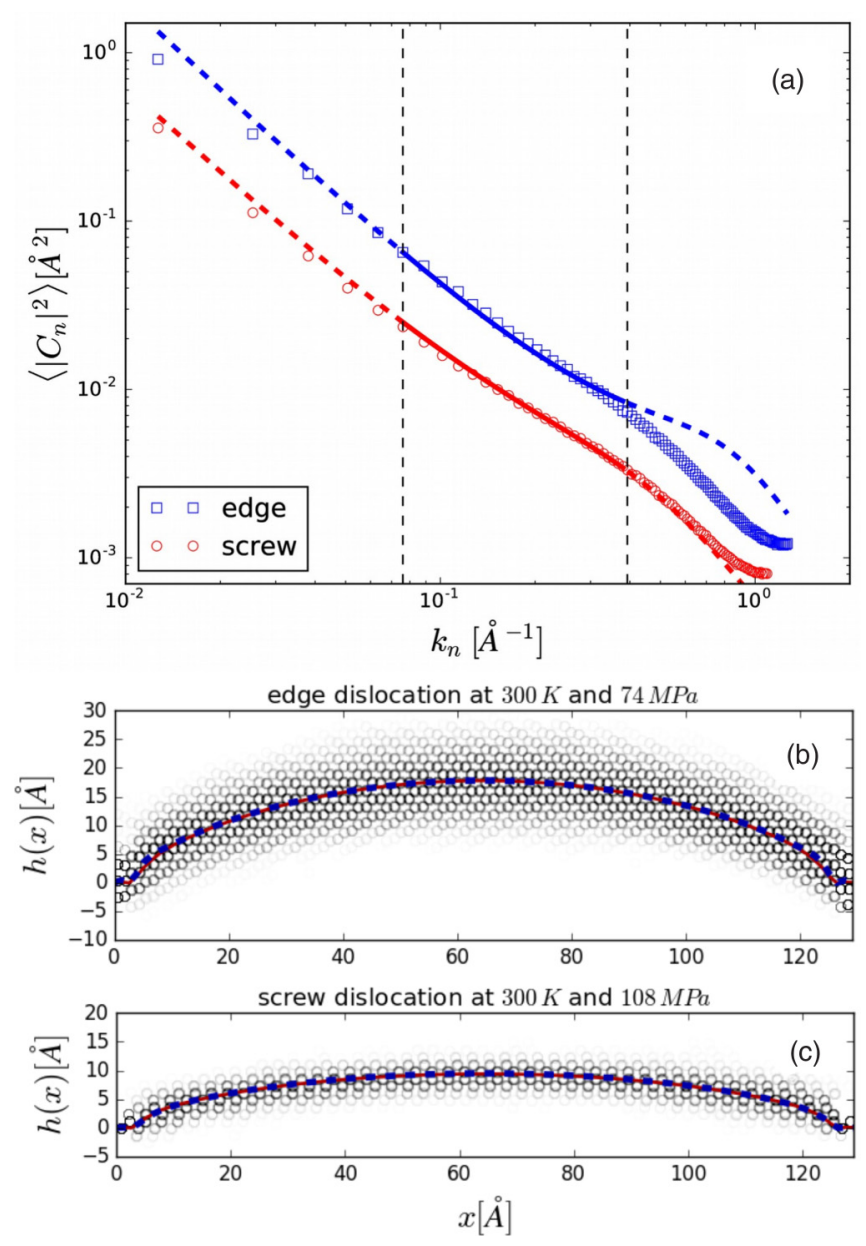

FIG. 6. (a) Fit of the power spectra obtained at $300 \mathrm{~K}$ by enforcing the same core parameter for the screw and edge characters. The fit is only performed for intermediate wave vectors in between the vertical dashed lines. (b)-(c) Comparison of bowing-out dislocation shape between atomistic simulations at $300 \mathrm{~K}$ and two sets of parameters: listed in Table II (red) obtained from the fit shown in (a) (dashed blue).

coefficients $\Gamma_{s}=0.119$ and $\Gamma_{e}=0.0297 \mathrm{eV} / \AA$ for the screw and edge characters, respectively. While this fit does not reproduce accurately the details of the fluctuations at small wavelengths $(<10 \AA)$, it reproduces satisfactorily the large wavelength regime. In addition, as shown in Figs. 6(b) and 6(c), the bow-out obtained with this set of parameters (shown with dashed blue lines) is essentially identical to the bow-out obtained with character-dependent core parameters (shown in red) because of the long wavelength involved in this bow-out.

Moreover, the $k$ dependence of the mass and drag coefficient pointed out in Sec. V cannot be accounted for in real space implementations of DD. Because DD is often used to investigate mesoscale dislocation microstructures, much larger than the dislocation lengths investigated here, this $k$ dependence could in principle be neglected. Then, limiting values at large wavelengths can be used $\left(M_{0}\right.$ and $B_{0}$ mentioned previously) to investigate these large dislocation assemblies. However, it is worth keeping in mind that these $M_{0}$ and $B_{0}$ parameters would not reproduce faithfully the dislocation 
dynamics when using DD to investigate mechanisms occurring on nanometric scales [4,24,41-43].

\section{CONCLUSION}

In conclusion, we propose here a method to analyze dislocation fluctuations by means of an analytical approach. Our analysis reveals the interplay between long-range elasticity and short-range core effects and shows the necessity to account for both to reproduce faithfully the dislocation behavior over a wide range of wavelengths. The reliability of the obtained parameters is tested by showing that they can reproduce very faithfully atomistic bow-out configurations. In addition, analysis of the time correlations of the fluctuations reveals a marked wavelength dependence of the dislocation dynamics. Finally, we discuss how to extract reliable parameters that can be used as input of higher-scale models such as dislocation dynamics.

We note that we have considered here the thermal fluctuations of isolated dislocations. They are not representative of the temperature-dependent plastic behavior at the microstructural scale, which is often controlled by collective effects [44-46]. Our work can, however, be used to parametrized quantitatively DD simulations that are the appropriate tool to study collective effects. Also, the analytical expression derived here [Eqs. (1)-(3)] can be used to investigate quantitatively other dislocation processes: natural extensions include thermally-activated processes such as kink-pair nucleation in friction-limited dynamics, the cross slip of screw dislocations or the thermally activated dislocation glide in dilute or high-entropy alloys.

\section{ACKNOWLEDGMENTS}

We would like to thank Emmanuel Clouet for fruitful discussions concerning the calculation of dislocation core energies from molecular static simulations. We acknowledge support of the Agence Nationale de La Recherche through Grant No. ANR-13-MERA-0001-02. P.A.G. gratefully acknowledges SR16000 supercomputing resources from the Center for Computational Materials Science of the Institute for Materials Research, Tohoku University. D.R. acknowledges support from LABEX iMUST (ANR-10-LABX-0064) of Université de Lyon (program "Investissements d'Avenir", ANR-11-IDEX-0007).
[1] J. Hirth and J. Lothe, Theory of Dislocations (McGraw-Hill, New York, 1968).

[2] E. Clouet, L. Ventelon, and F. Willaime, Phys. Rev. Lett. 102, 055502 (2009).

[3] D. Rodney, L. Ventelon, E. Clouet, L. Pizzagalli, and F. Willaime, Acta Mater. 124, 633 (2017).

[4] B. Szajewski, F. Pavia, and W. Curtin, Modell. Simul. Mater. Sci. Eng. 23, 085008 (2015)

[5] M. R. Gilbert, P. Schuck, B. Sadigh, and J. Marian, Phys. Rev. Lett. 111, 095502 (2013).

[6] T. D. Swinburne and M.-C. Marinica, Phys. Rev. Lett. 120, 135503 (2018).

[7] T. Vegge, T. Rasmussen, T. Leffers, O. B. Pedersen, and K. W. Jacobsen, Phys. Rev. Lett. 85, 3866 (2000).

[8] D. Caillard and J.-L. Martin, Thermally Activated Mechanisms in Crystal Plasticity (Elsevier, Amsterdam, 2003), Vol. 8.

[9] J. Friedel, Dislocations (Pergamon Press, Oxford, 1964).

[10] A. Granato, K. Lücke, J. Schlipf, and L. Teutonico, J. Appl. Phys. 35, 2732 (1964)

[11] C. Misbah, O. Pierre-Louis, and Y. Saito, Rev. Mod. Phys. 82, 981 (2010).

[12] J. J. Hoyt, M. Asta, and A. Karma, Phys. Rev. Lett. 86, 5530 (2001).

[13] M. Asta, J. J. Hoyt, and A. Karma, Phys. Rev. B 66, 100101(R) (2002).

[14] J. J. Hoyt, M. Asta, and A. Karma, Interface Sci. 10, 181 (2002).

[15] A. Karma and D. Tourret, Curr. Opin. Solid State Mater. Sci. 20, 25 (2016).

[16] A. Karma, Z. T. Trautt, and Y. Mishin, Phys. Rev. Lett. 109, 095501 (2012).

[17] D. Chen and Y. Kulkarni, J. Mech. Phys. Solids 84, 59 (2015).

[18] C. Sobie, L. Capolungo, D. McDowell, and E. Martinez, Acta Mater. 134, 203 (2017).
[19] M.-C. Miguel and M. Kardar, Phys. Rev. B 56, 11903 (1997)

[20] P. Moretti, M.-C. Miguel, M. Zaiser, and S. Zapperi, Phys. Rev. B 69, 214103 (2004).

[21] W. Cai, A. Arsenlis, C. Weinberger, and V. Bulatov, J. Mech. Phys. Solids 54, 561 (2006).

[22] A. Arsenlis, W. Cai, M. Tang, M. Rhee, T. Oppelstrup, G. Hommes, T. Pierce, and V. Bulatov, Modell. Simul. Mater. Sci. Eng. 15, 553 (2007).

[23] J. Marian and A. Caro, Phys. Rev. B 74, 024113 (2006).

[24] P.-A. Geslin, R. Gatti, B. Devincre, and D. Rodney, J. Mech. Phys. Solids 108, 49 (2017).

[25] T. D. Swinburne, S. L. Dudarev, S. P. Fitzgerald, M. R. Gilbert, and A. P. Sutton, Phys. Rev. B 87, 064108 (2013).

[26] P.-A. Geslin, B. Appolaire, and A. Finel, Phys. Rev. Lett. 115, 265501 (2015).

[27] See Supplemental Material at http://link.aps.org/supplemental/ 10.1103/PhysRevB.98.174115 for details of the calculations.

[28] J. Yin, D. Barnett, and W. Cai, Modell. Simul. Mater. Sci. Eng. 18, 045013 (2010).

[29] E. Clouet, Phys. Rev. B 84, 224111 (2011).

[30] E. Clouet, L. Ventelon, and F. Willaime, Phys. Rev. B 84, 224107 (2011).

[31] M. Boleininger, T. Swinburne, and S. Dudarev, Phys. Rev. Mater. 2, 083803 (2018).

[32] Y. Mishin, D. Farkas, M. Mehl, and D. Papaconstantopoulos, Phys. Rev. B 59, 3393 (1999).

[33] E. Clouet, "Babel", http://emmanuel.clouet.free.fr/Programs/ Babel/ (2010-2016).

[34] E. Clouet, Philos. Mag. 89, 1565 (2009).

[35] S. Plimpton, J. Comput. Phys. 117, 1 (1995).

[36] M. Gitterman, The Noisy Oscillator: The First Hundred Years, From Einstein Until Now (World Scientific, Singapore, 2005). 
[37] E. Bitzek, Atomistic simulation of dislocation motion and interaction with crack tips and voids, Ph.D. thesis, Universität Karlsruhe, 2006.

[38] E. Bitzek and P. Gumbsch, Mater. Sci. Eng. A 387-389, 11 (2004).

[39] V. Bulatov and W. Cai, Computer Simulations of Dislocations (Oxford University Press, Oxford, 2006), Vol. 3.

[40] B. Devincre, R. Madec, G. Monnet, S. Queyreau, R. Gatti, and L. Kubin, in Modeling Crystal Plasticity with Dislocation Dynamics Simulations: The 'MicroMegas' Code, edited by O. Thomas, A. Ponchet, and S. Forest (Presses de l'Ecole des Mines de Paris, Paris, 2011), pp. 81-100.
[41] R. Santos-Güemes, G. Esteban-Manzanares, I. Papadimitriou, J. Segurado, L. Capolungo, and J. LLorca, J. Mech. Phys. Solids 118, 228 (2018).

[42] E. Martinez, J. Marian, A. Arsenlis, M. Victoria, and J. Perlado, Philos. Mag. 88, 809 (2008).

[43] E. Martinez, J. Marian, and J. Perlado, Philos. Mag. 88, 841 (2008).

[44] M.-C. Miguel, A. Vespignani, S. Zapperi, J. Weiss, and J.-R. Grasso, Nature (London) 410, 667 (2001).

[45] J. Weiss, J.-R. Grasso, M.-C. Miguel, A. Vespignani, and S. Zapperi, Mater. Sci. Eng. A 309-310, 360 (2001).

[46] M.-C. Miguel, A. Vespignani, S. Zapperi, J. Weiss, and J.-R. Grasso, Mater. Sci. Eng. A 309-310, 324 (2001). 